\title{
Critical residues for histone acetylation by Gcn5, functioning in Ada and SAGA complexes, are also required for transcriptional function in vivo
}

\author{
Lian Wang, ${ }^{1}$ Lin Liu, ${ }^{1}$ and Shelley L. Berger ${ }^{2}$ \\ M olecular Genetics Program, The Wistar Institute, Philadel phia, Pennsylvania 19104 USA
}

\begin{abstract}
Several previously known transcription cofactors have been demonstrated in vitro recently to be histone acetyltransferases and deacetyltransferases, suggesting that remodeling of chromatin through histone acetylation plays a fundamental role in gene regulation. Clear evidence has not yet been obtained, however, to demonstrate that histone acetylation is required for gene activation in vivo. In this study we performed an alanine-scan mutagenesis through the HAT (histone acetyltransferase) domain identified previously by deletion mapping in recombinant yeast Gcn5. We identified multiple substitution mutations that eliminated completely Gcn5's ability to potentiate transcriptional activation in vivo. Strikingly, each of these mutations was also critical for free and nucleosomal histone acetylation by $\mathrm{Gcn} 5$ functioning within the native yeast HAT complexes, Ada, and SAGA. Moreover, the growth phenotypes of these mutations as measured by colony size and liquid growth assay closely tracked transcription and HAT activities. In contrast, mutations that did not affect in vivo function of $\mathrm{Gcn} 5$ were able to acetylate histones. These data argue strongly that acetylation is required for gene regulation by Gcn5 in vivo, and support previous arguments that nucleosomal histones are among the physiological substrates of acetylation by Gcn5.
\end{abstract}

Gene expression is a highly regulated process at the level of transcriptional activation. Recruitment of the transcriptional basal machinery by activators and cofactors has been shown to be an important component of gene regulation in vitro (Zawel and Reinberg 1995) and in vivo (Struhl 1995). Transcriptional activator proteins facilitate association of components of the basal machinery with the TATA box and start site of transcription (Triezenberg 1995). Under physiological conditions, DNA is complexed with histones forming nucleosomes that assemble into higher order chromatin (Wolffe 1992). Chromatin assembly strongly inhibits transcription (Owen-Hughes and Workman 1994; Paranjape et al. 1994), probably by blocking binding of transcription factors to their cognate DNA-binding sites. Therefore, in vivo, transcription factors must overcome the DNA access problem posed by nucleosomes. Moreover, genetic manipulations of histones confirm their important role in regulation of transcription (Grunstein 1990).

Both transcriptional activators and cofactors have critical roles in altering the repressive chromatin structure to promote binding of basal factors. Activation domains have been shown to be involved in remodeling chromatin (Svaren et al. 1994), and nucleosome struc-

\footnotetext{
${ }^{1}$ These authors contributed equally to this work.

${ }^{2}$ Corresponding author.

E-MAIL berger@wista.wistar.upenn.edu; FAX (215) 898-0063.
}

tural changes occur in the absence of transcription sug gesting that activators may induce transcription by causing chromatin changes before initiation (Fascher et al. 1993). Transcriptional cofactors al so activate or repress transcription by destablizing or stablizing the repressive nucleosome structure (Kingston et al. 1996). For example, the SWI/SN F family of protein complexes activates transcription by disrupting the nucleosome structure in an ATP-dependent manner to increase the binding of transcription factors (Cote et al. 1994; Peterson et al. 1994).

Nucleosomes are composed of $\sim 146$ bp of DNA wrapped around the histone octamer, consisting of two molecules each of the histones $\mathrm{H} 2 \mathrm{~A}, \mathrm{H} 2 \mathrm{~B}, \mathrm{H} 3$, and $\mathrm{H} 4$ (Wolffe 1992). Each core histone contains a gl obular domain and extended basic amino-terminal tail that stabilizes histone octamer association with DNA through ionic interactions. Covalent modifications of nucleosomal histones have been correlated with modulation of chromatin structure and function (Bradbury 1992). These modifications include phosphorylation, ubiquitination and, in particular, acetylation of $\epsilon$ amino groups of lysines in the histone tails. Acetylation is associated with both gene activation (hyperacetylation) and gene silencing (hypoacetylation) (for review, see Loidl 1994; Turner and O'N eill 1995). A cetylation of histones increases the affinity of transcription factors for nucleosomal DNA (Lee et al. 1993; Vettese-Dadey et al. 1996). 
The recent identification of histone acetyltransferase (HAT) and histone deacetylase (HDAC) enzymes as previously recognized transcripti on cofactors (Pazin and Kadonaga 1997; Wade and Wolffe 1997) suggests a direct mechanistic connection between the activity of transcription factors and changes in chromatin structure that precede initiation. The first nuclear HAT enzyme, p55, was purified and cloned from Tetrahymena, and was found to be closely rel ated to yeast Gcn5 (Brownell et al. 1996), a known transcription coactivator/adaptor (Guarante 1995). The ability of yeast Gcn5, and its binding partner yeast Ada2, to associate with activation domains (Barlev et al. 1995; Chiang et al. 1996; Silverman et al. 1994) and with the TATA-binding protein (TBP; Barlev et al. 1995), suggests that acetylation of nucl eosomal histones through activation domain recruitment of Gcn5 may be a fundamental and novel feature of gene regulation. Supporting this notion were subsequent revel ations that several other transcription coactivators/adaptors possess intrinsic HAT activity, including additional Gcn5 family members [hGcn5 (Wang et al . 1997; Yang et al. 1996) and P/CAF (Y ang et al. 1996)] as well as unrelated CBP/p300 (Bannister and Kouzarides 1996; Ogryzko et al. 1996) and TAF 250 (Mizzen et al. 1996).

Conversely, the first HDAC was identified (T aunton et al. 1996) and strikingly, it also was a previously known transcription factor, Rpd3 (Vidal and Gaber 1991), possessing transcriptional repression activity. Several other transcriptional repressors have been shown to be HDACs, including Sin3 (Hassig et al. 1997; Kadosh and Struhl 1997; Laherty et al. 1997; Zhang et al. 1997) and the corepressor N-CoR/SM RT (Alland et al. 1997; Heinzel et al. 1997). Despite these provocative observations, however, direct evidence has not yet been obtained that acetylation or deacetylation of histones has a direct role in gene regulation.

Although recombinant proteins have been tested for histone acetylation and deacetylation activity, recent studies demonstrate that, in vivo, the HATs (Grant et al. 1997; Horiuchi et al. 1997; Sal eh et al . 1997) and HDACs (Rundlett and Grunstein 1996; Zhang et al. 1997) function as components of high-molecular-weight complexes. For example, yeast Gcn5 is the catalytic HAT subunit present in two complexes, termed $A$ da and SAGA (Spt, A da, Gcn5 acetyltransferase; Grant et al. 1997). The A $\bar{d} a$ complex al so contains Ada2 and Ada3, which were similarly isolated as suppressors of growth toxicity caused by overexpression of the potent chimeric activator composed of the yeast Gal4 DN A-binding domain fused to the herpes virus VP16 activation domain (Berger et al. 1992; Piña et al. 1993; M arcus et al. 1994). In addition to the Ada proteins, the SAGA complex contains Spt7 (Gansheroff et al . 1995) and Spt20 (Roberts and Winston 1996) proteins, which were isol ated as suppressors of Ty element insertions. Interestingly, Spt20/A da5 was identified in both Ada and Spt genetic selections (Marcus et al. 1996; Roberts and Winston 1996) and is present in the SAGA complex (Grant et al. 1997). The importance of determining acetylation activity within these native complexes is illustrated by the observation that recombinant yeast Gcn5 acetylates only free histones, whereas, in association with A da and SA GA complexes, Gcn5 acetylates nucleosomal histones (Grant et al. 1997). In addition, genetic studies demonstrate that SA GA contains functions involved in transcriptional activation distinct from those mediated by the Gcn5/ Ada2/Ada3 group (Roberts and Winston 1997), underscoring further the importance of testing HAT activity in the context of native complexes.

The boundaries of the HAT domain in recombinant yeast Gcn5 were mapped and the region was shown to be required for Gcn5's role in transcriptional activation in vivo (Candau et al. 1997). This region of the Gcn5 family members Tetrahymena p55 (Brownell et al. 1996), yeast Gcn5 (Georgakopoulos and Thireos 1992), human Gcn5 (Candau et al. 1996), and human P/CAF (Yang et al. 1996) is remarkably conserved in primary sequence (Brownell et al. 1996). Certain sequence motifs have been identified in these HAT domains and are found in other acetyltransferases (Reifsnyder et al . 1996; N euwal d and Lansman 1997). Paradoxically, the other transcriptional coactivators possessing intrinsic HAT activity, $\mathrm{CBP} / \mathrm{p3} 300$ and $\mathrm{TAF}_{11} 250$, display no obvious sequence homology within the putative HAT domain compared with the Gcn5 family (Bannister and Kouzarides 1996; Mizzen et al. 1996; Ogryzko et al. 1996). In fact, recent data demonstrates that, in addition to histones, p300 acetylates the transcriptional activator p53, and, surprisingly, with nearly equal strength (Gu and Roeder 1997).

The data described above rai se important questionswhat are the critical residues for acetylation of histone substrates in Gcn5 and are these residues required for Gcn5's role in transcriptional activation? If so, this would argue that first, acetylation is a critical and direct determinant of gene regulation in vivo, and second, that histones are likely to be a critical physiological substrate. In the current study, we have undertaken al aninescan mutagenesis to address these issues. The role of specific mutations was tested on Gcn5 functioning within the context of native yeast Ada and SAGA compl exes. Our results demonstrate clearly that critical residues for histone acetylation in Gcn5 are al so required for its in vivo function in growth and as a transcription cofactor. These data provide compelling evidence that acetylation by Gcn5 is crucial for transcriptional activation in vivo.

\section{Results}

Construction of alanine-scan mutants within the putative HAT domain of Gcn5

The four Gcn5 family members that have been identified are yeast Gcn5, human Gcn5, human P/CAF, and Tetrahymena $\mathrm{p55}$. Although the overall sequence identity between the four proteins is $<40 \%$, the amino acid sequence in the putative HAT domain is $67 \%$ identical and $82 \%$ similar (Fig. 1A). The primary structural similarity is reflected in conservation of function between the human and yeast HAT domains, whereas no other domain 
Figure 1. Amino acid sequence within the putative HAT domains of the Gcn5 family. (A) Sequence comparison between the putative HAT domains of yeast Gcn5, human P/CAF, human Gen5 and Tetrahymena p55. The protein sequence within the putative HAT domains are shown by single amino acid letter-code. N umbers at left indicate amino acid residues. The highly conserved regions I-IV (Brownell et al. 1996) are indicated at the top. Solid boxes indicate amino acid identity and shaded boxes indicate similarity. (B) Y east Gcn5 substitution mutants created in the study. The substitution mutants are $\mathbf{B}$ named according to the group of amino acids mutated to alanine, each of which is indicated by brackets.
$\mathbf{A}$

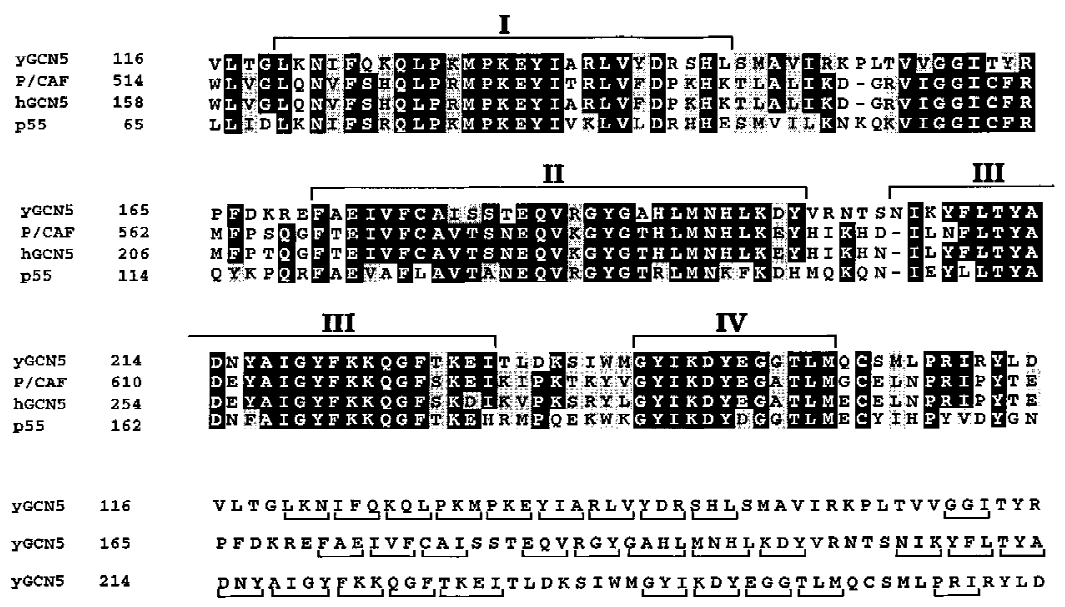

of human Gcn5 is capable of function in yeast (Wang et al. 1997). The HAT domain of Gcn5 can be divided into four subdomains, I, II, III, and IV (Brownell et al. 1996), based on exceptionally strong sequence identity between all family members (Fig. 1A).

To identify critical amino acids for HAT enzymatic activity and to determine whether HAT activity is crucial for Gcn5's role in transcription and growth, an alanine-scan mutagenesis was performed. Three or four adjacent amino acids were grouped (indicated as three or four amino acid letter code) and mutagenized to alanine (Fig. 1B) using site-directed mutagenesis. The mutagenic oligonucleotides (Table 1) contained a Pstl restriction site allowing initial positive identification of each mutant, which was confirmed by DN A sequencing. Thirtyone substitution mutants were created and their phenotypes studied.

\section{Growth complementation by Gcn5 substitution mutants in the gcn5 ${ }^{-}$strain}

The ability of the mutant GCN5 genes to complement phenotypes caused by GCN5 disruption in yeast was tested. Yeast lacking GCN5 grow poorly on minimal synthetic media, and transformation of wild-type GCN 5 completely complements the growth defect (Georgakopoulos and Thireos 1992; Marcus et al. 1994). Complementation by the Gcn5 substitution mutants was tested by transforming plasmids bearing each of the mutant genes into the gcn5 ${ }^{-}$strain and assessing growth using colony size (Fig. 2A) and doubling time (Fig. 2B). The mutant genes were expressed using the natural promoter and terminator of GCN5 to obtain normal levels of protein expression. The majority of Gcn5 substitution mutants (19 of the 30 mutants shown, as well as GGI located between regions I and II) complemented growth as well as wild-type Gcn5, as the yeast transformants yielded colony sizes that were indistinguishable from wild type (Fig. 2A). Several mutants (YIA, RGY, DNY, KDY242, and TLM) displayed variable intermediate colony sizes, ranging between that achieved by yeast bearing wild-type GCN 5 or a disruption of the gene (Fig. 2A). Six mutants (KQL, PKM, FAE, KDY 196, AIGY, and FKK) completely failed to complement the colony growth defect in the gcn5 ${ }^{-}$strain (Fig. 2A).

Relative growth phenotypes were quantitated by measuring doubling times in liquid minimal growth media. The Gcn5 mutants that exhibited the poorest growth in the colony assay were analyzed, as well as several representative mutants that exhibited wild-type growth. The results paralleled the colony assays because the most defective mutants (KQL, PKM , FAE, KDY 196, AIGY, and FKK) grew at essentially the rate of the gcn $5^{-}$strain, and the wild-type mutants grew comparably with GCN5 ${ }^{+}$ (Fig. 2B). Furthermore, the RGY mutant that showed intermediate-sized colonies was also intermediate in its rate of doubling (Fig. 2B). Based on the two growth complementation assays, we tentatively grouped the mutants into three classes-those that were essentially wild type, those that exhibited significant loss of growth complementation, and those that were completely defective.

The failure of Gcn5 mutants to complement growth may be caused by defective function of the Gcn5 substitution mutants, or alternatively, by structural defects in the protein causing either instability or inability to form requisite protein-protein interactions in vivo. To address the question of protein stability, the expression levels of the mutant $\mathrm{G} c n 5$ proteins in yeast were determined relative to wild-type Gcn5. Gcn5 was expressed under control of the galactose-inducible GAL1-10 promoter and intracellular protein expression levels were determined by Western analysis (Fig. 3, top). Although there was variation in protein levels, all Gcn5 mutants were present at levels comparable with wild-type Gcn5, and, in particular, all mutants displaying partial or complete defects in the growth assay were detectable in vivo (Fig. 3, top).

To determine the structural integrity of the mutant proteins in vivo, we tested interaction with Ada2, as our previous results demonstrated that $A$ da2 interaction is critical for Gcn5 function in vivo (Candau et al. 1997). 
Table 1. Sequences of mutagenic oligonucleotides

\begin{tabular}{|c|c|c|}
\hline Name ${ }^{a}$ & Amino acid & Sequence of oligonucleotides \\
\hline LKN & 120 & GATGGTCCTAACTGGAGCTGCAGCCATTTTTCAAAAGCAATTACC \\
\hline IFQ & 123 & CTAACTGGATTAAAAAACGCTGCAGCAAAGCAATTACCAAAAATG \\
\hline KQL & 126 & GGATTAAAAAACATTTTTCAAGCTGCAGCACCAAAAATGCCC \\
\hline PKM & 129 & GGATTAAAAAACATTTTTCAAAAGCAATTAGCTGCAGCGCCCAAAGAATACATTGCC \\
\hline PKE & 132 & CAATTACCAAAAATGGCTGCAGCATACATTGCCAGGTTAG \\
\hline RLV & 135 & ATGCCCAAAGAAGCTGCAGCCAGGTTAGTCTATG \\
\hline YIA & 138 & CAAAGAATACATTGCCGCTGCAGCCTATGATCGAAGTCATC \\
\hline YDR & 141 & GCCAGGTTAGTCGCTGCAGCAAGTCATCTTTCCATG \\
\hline SHL & 144 & GTTAGTCTATGATCGAGCTGCAGCTTCCATGGCTGTCATTAGG \\
\hline GGI & 159 & CGATAAGAGAGAAGCCGCAGCAATTGTTTTCTGTGCC \\
\hline FAE & 171 & CCATTGACTGTCGTAGCTGCAGCAACATATCGACCTTTCG \\
\hline IVF & 174 & GAATTCGCAGAAGCTGCAGCCTGTGCCATCAGTTCG \\
\hline CAI & 177 & CGCAGAAATTGTTTTCGCTGCAGCCAGTTCGACGGAACAG \\
\hline EQV & 183 & GCCATCAGTTCGACGGCTGCAGCACGCGGTTATGGTGCG \\
\hline RGY & 186 & GGAACAGGTAGCTGCAGCTGGTGCGCATC \\
\hline GAHL & 189 & GGTACGCGGTTATGCTGCAGCTGCAATGAATCACTTAAAAGAC \\
\hline MNHL & 193 & GGTTATGGTGCGCATCTAGCGGCTGCAGCAAAAGACTATGTTAG \\
\hline KDY 196 & 196 & GAATCACTTAGCTGCAGCTGTTAGAAATACC \\
\hline NIK & 205 & GTTAGAAATACCTCGGCTGCAGCATATTTTTTGACATATGC \\
\hline YFL & 208 & CCTCGAACATAAAAGCTGCAGCGACATATGCAGATAATTAC \\
\hline TYA & 211 & CGAACATAAAATATTTTTTGGCTGCAGCAGATAATTACGC \\
\hline DNY & 214 & TATTTTTTGACATATGCAGCTGCAGCCGCTATTGGATACTTT \\
\hline AIGY & 217 & GCAGATAATTACGCTGCTGCAGCCTTTAAAAAGCAAGGC \\
\hline FKK & 221 & TACGCTATTGGATACGCTGCAGCGCAAGGCTTCACTAA \\
\hline QGF & 224 & CGCTATTGGATACTTTAAAAAGGCTGCAGCCACTAAAGAAATCACGTTGG \\
\hline TKEI & 227 & AAAAAGCAAGGCTTCGCTGCAGCAGCCACGTTGGATAAAAG \\
\hline GYI & 239 & GTATATGGATGGCTGCAGCTAAAGATTATGAAGG \\
\hline KDY 242 & 242 & GGATGGGATATATTGCTGCAGCTGAAGGTGGT \\
\hline EGG & 245 & GGATATATTAAAGATTATGCTGCAGCTACGCTGATGCAATG \\
\hline TLM & 248 & GATTATGAAGGTGGTGCTGCAGCGCAATGTTCTATGTTACC \\
\hline PRI & 256 & GCAATGTTCTATGTTAGCTGCAGCACGATATTTGGACGC \\
\hline
\end{tabular}

aThe name of each mutant represents the amino acids mutagenized.

${ }^{\text {b }}$ The position of the first amino acid mutagenized in each mutant is indicated.

Yeast ADA2 was fused to glutathione-S-transferase (GST ) foll owed by cotransformation into yeast of expression plasmids bearing GST-ADA2 and GCN5. Y east extracts were incubated with GST beads and the binding of wild-type Gcn5 or Gcn5 mutants to GST-Ada2 was determined by immunoblot. The Gcn5 mutants were able to bind to Ada2 and importantly, mutants defective for growth complementation also interacted with Ada2 (Fig. 3, bottom). Overall, the yeast expression experiments and Ada2-interaction results indicate that the defective phenotypes of the substitution mutants were not due to global structural defects.

Transcriptional activation in the presence of Gcn5 mutants in the gcn5 ${ }^{-}$strain

The ability of Gen5 substitution mutants to support transcriptional activation in the gcn $5^{-}$strain was tested. Full transcriptional potency by certain transcriptional activators requires Gcn5 (Georgakopoulos and Thireos 1992; Marcus et al. 1994). Two chimeric activators were tested. The first was the yeast Gal4 DNA-binding domain fused to the potent activation domain of herpes simplex virus activator VP16 (Gal4-VP16), and the sec- ond was the bacterial LexA DNA binding domain fused to the activation domain from the yeast activator Gcn4 (LexA-Gcn4). The expression reporters were bacterial LacZ driven by yeast Gal4 DNA-binding sites or bacterial LexA DNA-binding sites, and transcriptional potency was determined by quantitative $\beta$-gal actosi dase assays.

Both Gal4-VP16 and LexA-Gcn4 transcriptional activity were reduced in the GCN5 deletion strain to $9 \%$ of wild type (Fig. 4). The Gcn5 substitution mutants divided into three classes. Most Gcn5 mutants were capable of potentiating Gal4-VP16 or LexA-Gcn4 activity to approximately the level observed in the $\mathrm{GNN}^{+}$strain (Fig. 4). Several mutants that displayed partial complementation in the growth assay also showed an intermediate level of activity in potentiating transcriptional activation by both activators (Fig. 4). The six mutants (KQL, PKM, FAE, KDY 196, AIGY, and FKK) that were unable to complement the growth defect of gen5-, also failed to complement gcn5-function in the transcription assay for either activator (Fig. 4).

Therefore, the majority of the Gcn5 substitution mutants had similar phenotypes exhibited by wild-type Gcn5 in growth and transcription assays (hereafter re- 
Wang et al.

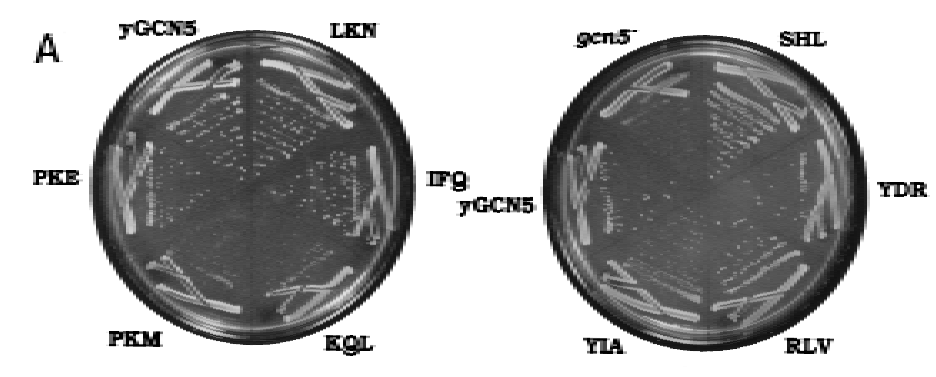

\begin{tabular}{c|c} 
B $\begin{array}{c}\text { Gcr5 } \\
\text { mutant }\end{array}$ & $\begin{array}{c}\text { generation } \\
\text { Lime } \\
\text { (hours) }\end{array}$ \\
\hline WT & 2.15 \\
vector & 4.50 \\
LKN & 2.50 \\
PKE & 2.25 \\
YDR & 2.20 \\
GAHL & 2.31 \\
GYI & 2.67 \\
KGL & 5.40 \\
PKM & 4.40 \\
FAE & 4.90 \\
RGY & 3.45 \\
KDYI96 & 4.30 \\
AlGY & 4.40 \\
FKK & 4.50 \\
\hline
\end{tabular}
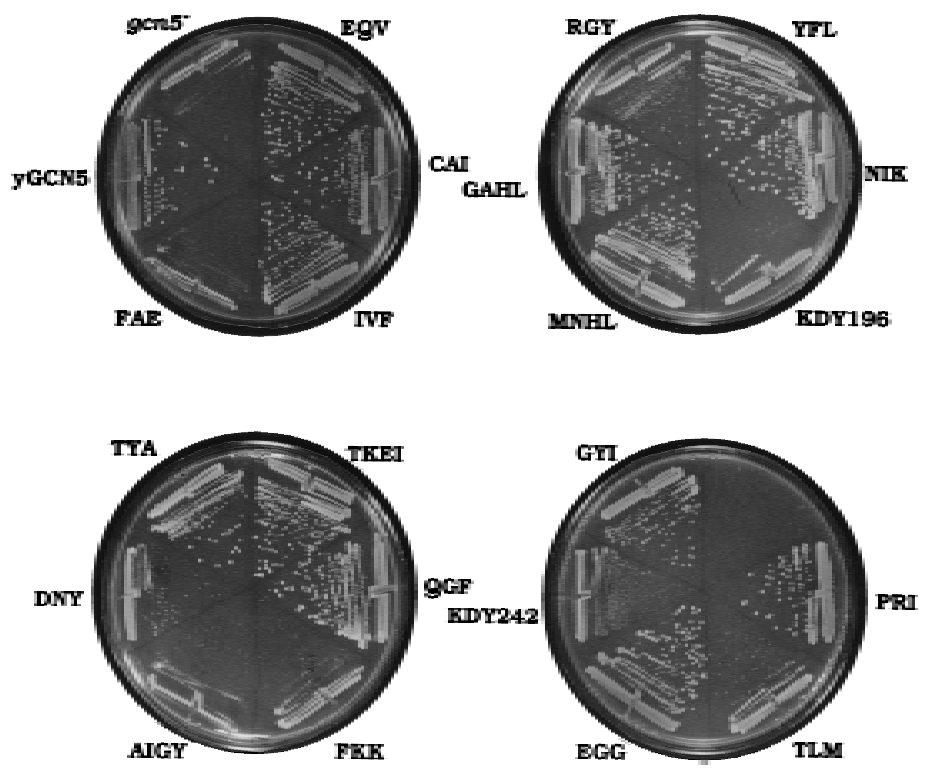

Figure 2. Growth phenotypes of GCN5 substitution mutants in the gen5 ${ }^{-}$strain. Wild-type GCN5, each of the GCN5 mutants, or vector alone were introduced into gen $5^{-}$strain. (A) Colony growth assay. Transformants were streaked onto synthetic minimal medium. Colony growth was assessed after 2 days of growth at $30^{\circ} \mathrm{C}$. (B) Doubling time of the Gcn5 substitution mutants. Aliquots of cultures in liquid synthetic minimal medium were taken every $2 \mathrm{hr}$ and the $A_{600}$ of the cultures was measured. The generation time was calculated as the OD doubling time during exponential growth.

ferred to as wild-type mutants; light gray bars in Fig. 4). The assays identified six Gcn5 mutants (KQL, PKM, FAE, KDY 196, AIGY, and FKK) that profoundly reduced Gcn5 function in vivo ("defective" mutants; black bars in Fig. 4). In addition, several mutants (YIA, RGY, DNY, KDY 242, and TLM ) displayed various degrees of partial function and are likely to represent important, but not crucial, residues for Gcn5 activity ("intermediate" mutants; dark gray bars in Fig. 4).

\section{Dominant-negative effect of Gcn5 mutants}

Mutations in catalytic site residues may cause mutant proteins to exert dominant-negative effects when overexpressed in vivo. Dominant-negative phenotypes are likely caused by the mutant enzyme competing with the wild-type counterpart for association with other proteins or assembly into protein complexes. To determine whether the phenotypical ly defective Gcn5 mutants exert a dominant defective growth effect in vivo, each was overexpressed in yeast containing normal levels of wildtype Gcn5.

Overexpression of wild-type Gcn5 itself caused yeast to grow slowly compared with transformed vector (Fig. 5), perhaps attributable to sequestration of associated proteins (Gill and Ptashne 1988). Overexpression of the wild-type Gcn5 mutants showed similar phenotypes as wild-type Gcn5 (data not shown) and were therefore judged not to be dominant-negative. Overexpression of most defective Gcn5 mutants caused even stronger growth inhibition relative to wild-type Gcn5 (Fig. 5).

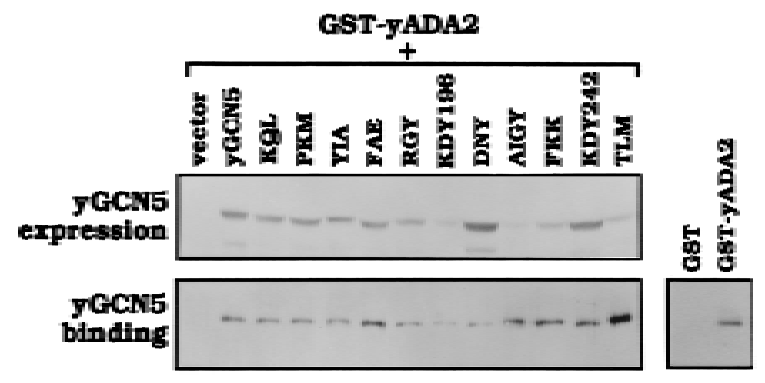

Figure 3. Stability and $A d a 2$ interaction of $G \mathrm{cn} 5$ substitution mutants in yeast. Wild-type GCN5, mutant GCN5, or vector al one were cotransformed with GST-ADA2, and proteins were induced using the GAL1-10 promoter. (Top) G cn5 protein levels in the yeast extracts were determined by Western blot analysis using Gcn5 antisera. (Bottom left) The yeast extracts were incubated with GST beads to allow GST-Ada2 to bind. Wild-type or mutant Gcn5 bound to GST-Ada2 was determined by Western analysis using Gcn5 antiserum. (Bottom right) Co-expression of wild-type Gcn5 with GST alone was used as a negative control. 


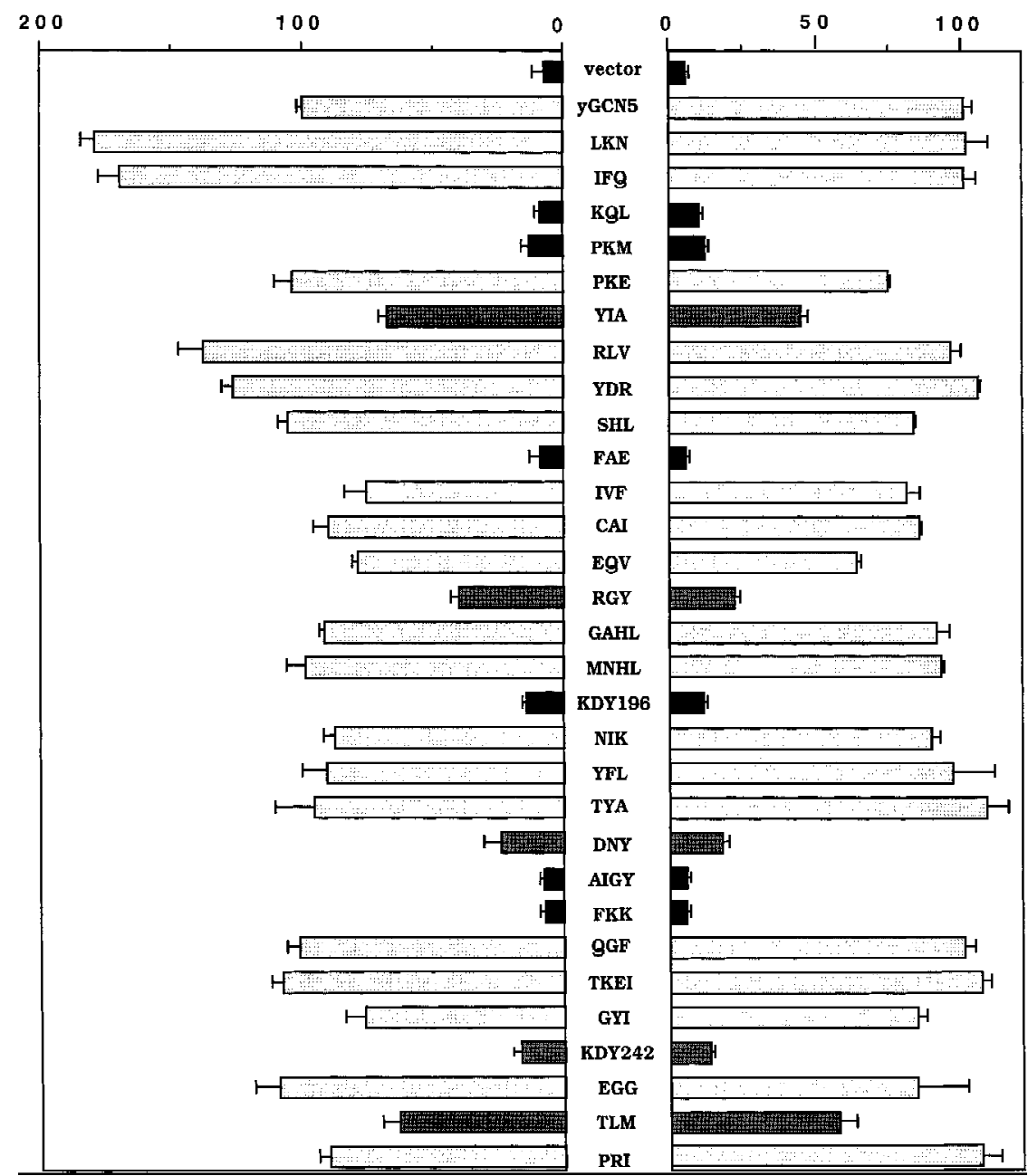

Figure 4. Transcriptional activation in the presence of $\mathrm{Gcn} 5$ substitution mutants. GAL4-VP16 (left panel) and LexAGCN 4 (right panel) were cotransformed with wild-type GCN5, each of the GCN5 mutants or vector al one, al ong with the appropriate LacZ reporters. $\beta$-Gal activities of the mutants are shown as a percentage of wild-type Gcn5, which was set at $100 \%$. (Solid bars) M utants that were defective in the growth and transcription assays; (dark gray bars) mutants having intermediate phenotypes; (light gray bars) mutants having activity similar to wild-type Gen5. Error bars represent the standard error about the mean from three independent experiments.
This was true both of mutants that were completely (KQL, PKM, FAE, AIGY, and FKK) or partially (YIA, RGY, and KDY 242) defective (Fig. 5). Two defective mutants (completely defective KDY 196 and partially defective TLM) exhibited a similar phenotype as wild-type Gcn5 (Fig. 5) and therefore were not dominant-negative. The mutants are likely to exert their dominant-negative effects by incorporation into normal protein complexes (Grant et al . 1997) because first, they are vastly overexpressed relative to wild-type Gcn5, and second, they interact normally with Ada2 (Fig. 3) and within native complexes (Fig. 6B,C) in the gcn5 ${ }^{-}$strain where wildtype Gcn5 is not present.

Therefore, two lines of evidence indicate that the
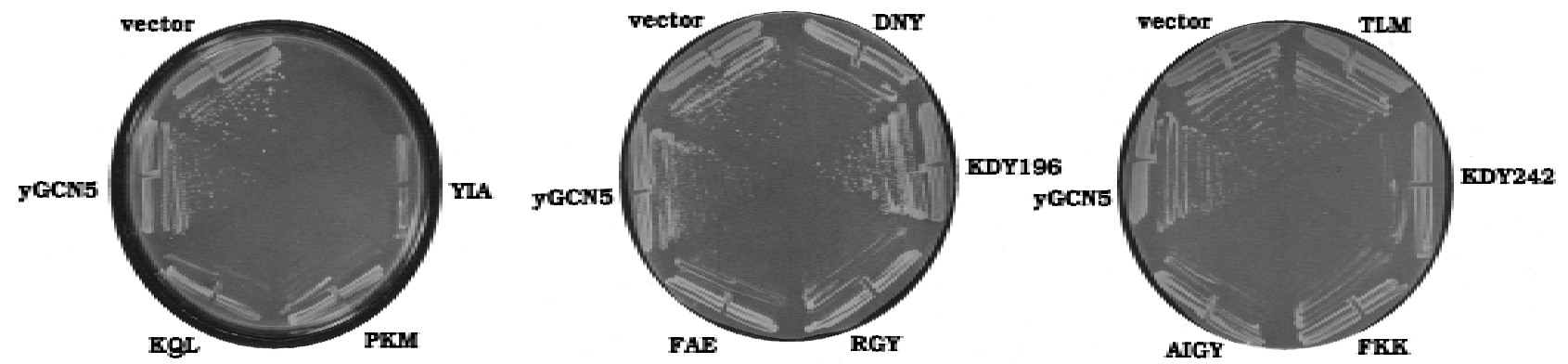

Figure 5. Dominant-negative effects of Gcn5 HAT mutants. Wild-type GCN5, the GCN 5 mutants, or vector al one were transformed into yeast strain bearing wild-type GCN5. The transformants were restreaked onto gal actose plates to induce expression of transformed $\mathrm{G} c n 5$ proteins and grown at $25^{\circ} \mathrm{C}$ for 6 days. 
Gcn5 mutants displaying defects in vivo are functional. First, all of the defective mutants possess structural integrity as manifested by their ability to interact with Ada2 (Fig. 4), and second, the majority of the defective mutants exhibit dominant negativity in vivo (Fig. 5).

Histone acetylation by $\mathrm{Gcn} 5$ substitution mutants within Ada and SAGA complexes

Previous studies have tested HAT activity of recombinant proteins. The HATs and HDACs are active within high-molecular-weight complexes, however, which influences their specificity (Rundlett and Grunstein 1996; Grant et al. 1997). To directly assay whether the defective phenotypes of the substitution mutants were caused by loss of ability to acetylate histones, HAT activity of the Gcn5 substitution mutants was tested in the context of high-molecular-weight native HAT complexes (Grant et al. 1997). Two high-molecular-weight native yeast complexes have been identified. Both contain Gcn5 as the HAT catal ytic subunit, and specifical ly acetylate histone $\mathrm{H} 3$ and, to a lesser extent, histone $\mathrm{H} 2 \mathrm{~B}$. The smaller complex $(800 \mathrm{kD})$ was named $\mathrm{Ada}$ and the larger one (2 MD) SAGA, based on the identity of their constituent proteins (Grant et al. 1997). We partially purified Ada and SAGA complexes from yeast expressing the Gcn5 substitution mutants, to determine whether the mutations affected the HAT activity within these physiological HAT complexes.

Wild-type Gcn5 or the substitution mutants were transformed into the gcn5 $5^{-}$strain using vectors to express normal levels of protein. Whole-cell extracts were prepared from 26 out of the 31 mutants created and were fractionated through $\mathrm{Ni}^{2+}$ agarose and $\mathrm{MonoQ}$ ion exchange resins (Grant et al. 1997). HAT activities were determined on free histone substrates for 22 out of these 26 extracts, which were determined by Western analysis to have nearly normal Gcn5/Ada2 protein levels in both the Ada and SAGA complexes (see below). These included 11 mutants that were partially or completely defectivein growth (Fig. 2) and transcription (Fig. 4), as well as 11 wild-type mutants.

The HAT activity of the peak SAGA fraction from wild-type or mutant Gcn5 was then determined by liquid HAT assay quantitation of $\left[{ }^{3} \mathrm{H}\right.$ ]acetyl group incorporation into histones using scintillation counting and visualization of histones by PAGE and fluorography. SAGA fractions derived from strains bearing wild-type mutant Gcn5 were able to acetylate histones, as shown by the examples of LKN, PKE, YDR, GAHL, and GYI (Fig. 6A). Four of these had levels as high as, or nearly as high as, wild-type Gcn5 (LKN, PKE, YDR, and GYI) as quantitated by scintillation counting (Fig. 6A, top) and confirmed by visualization of $\left[{ }^{3} \mathrm{H}\right]$ lacetyl incorporation into histone H3 (Fig. 6A, middle). These mutant complexes displayed Gon5 and Ada2 protein levels that were comparable with wild-type Gcn5 (Fig. 6B, top). The remaining wild-type mutant (GAHL) possessed $>50 \%$ of wild-type activity (Fig. 6A) and was found to be somewhat reduced in protein level compared with wild-type
Gcn5 and the other wild-type mutants (Fig. 6B, top; data not shown). In addition, visualization of $\left[{ }^{3} \mathrm{H}\right]$ acetyl incorporation into histone $\mathrm{H} 3$ catalyzed by SAGA bearing GAHL indicated clearly higher activity than the defective Gcn5 mutants (Fig. 6A, middle).

The six severely defective mutants (KQL, PKM, FAE, KDY 196, AIGY, and FKK), as well as partially defective RGY, displayed greatly reduced ability to acetylate histones, using both the quantitative assay as well as histone visual ization (Fig. 6A, top and middle). Five of the extracts showed background levels of HAT activity ( $25 \%$ of wild-type level). The level of acetylation by SA GA prepared from the defective FKK mutant was $45 \%$ of wild type in the quantitative assay, but was nearly as low as background levels by $\left[{ }^{3} \mathrm{H}\right.$-histone fluorography. (As is obvious in Fig. 6, we consistently observe that fluorography is a more sensitive measure of acetylation than scintillation quantitation.) The protein levels of both Ada2 and the defective mutant G cn5 proteins were variable, but the levels were similar to those in the strain expressing wild-type Gcn5 (Fig. 6B, bottom). Therefore, consistent with the stability assay (Fig. 3), the defects of these mutants were not attributable to lack of protein expression, or importantly, inability to be incorporated into the SAGA complex.

We then determined the HAT activity of the A da compl exes using the identical set of mutant Gcn5 proteins as used for assay of the SAGA complexes. In the case of the A da compl ex only $\left[{ }^{3} \mathrm{H}\right]$ histone-H 3 fluorography was used to determine HAT activity, as a strong overlapping Gcn5-independent histone $\mathrm{H} 4$ acetylation activity (Grant et al. 1997; see Fig. 7) obscured quantitation by scintillation counting of the Gcn5-dependent histone H3 activity. As observed for the SAGA complex, each of the wild-type mutants displ ayed activities comparable with wild-type Gcn5, whereas HAT activity by the defective mutants was not detectable (Fig. 6A, bottom). Western analysis of Ada2 and Gcn5 in the Ada complex (Fig. 6C) of the wild-type (Fig. 6C, top) and defective (Fig. 6C, bottom) Gcn5 mutants indicated comparable levels of protein with wild-type Gcn5. N ote that the Ada complex containing the GAHL mutant eluted primarily in fraction 20 from the MonoQ column (Fig. 6C, top left), whereas the remainder of the wild-type mutants eluted in a broader peak covering fractions from 20 (Fig. 6C, top left) to 24 (Fig. 6C, top right).

Overall, the results demonstrate a clear correlation between complementation of function by the Gon 5 substitution mutants in the gen5 ${ }^{-}$strain (Figs. 2 and 4) and acetylation of histones (Fig. 6). Importantly, HAT activity by the mutant $\mathrm{Gcn} 5$ proteins was similar in both the Ada and SAGA complexes, such that mutants that possessed wild-type levels of activity in the Ada complex were al so competent in the SA GA complex and mutants that were defective in Ada were al so defective in SAGA.

Acetylation of nucleosomal histones by Gcn5 substitution mutants

Both the SAGA and Ada complexes acetylate nucleo- 

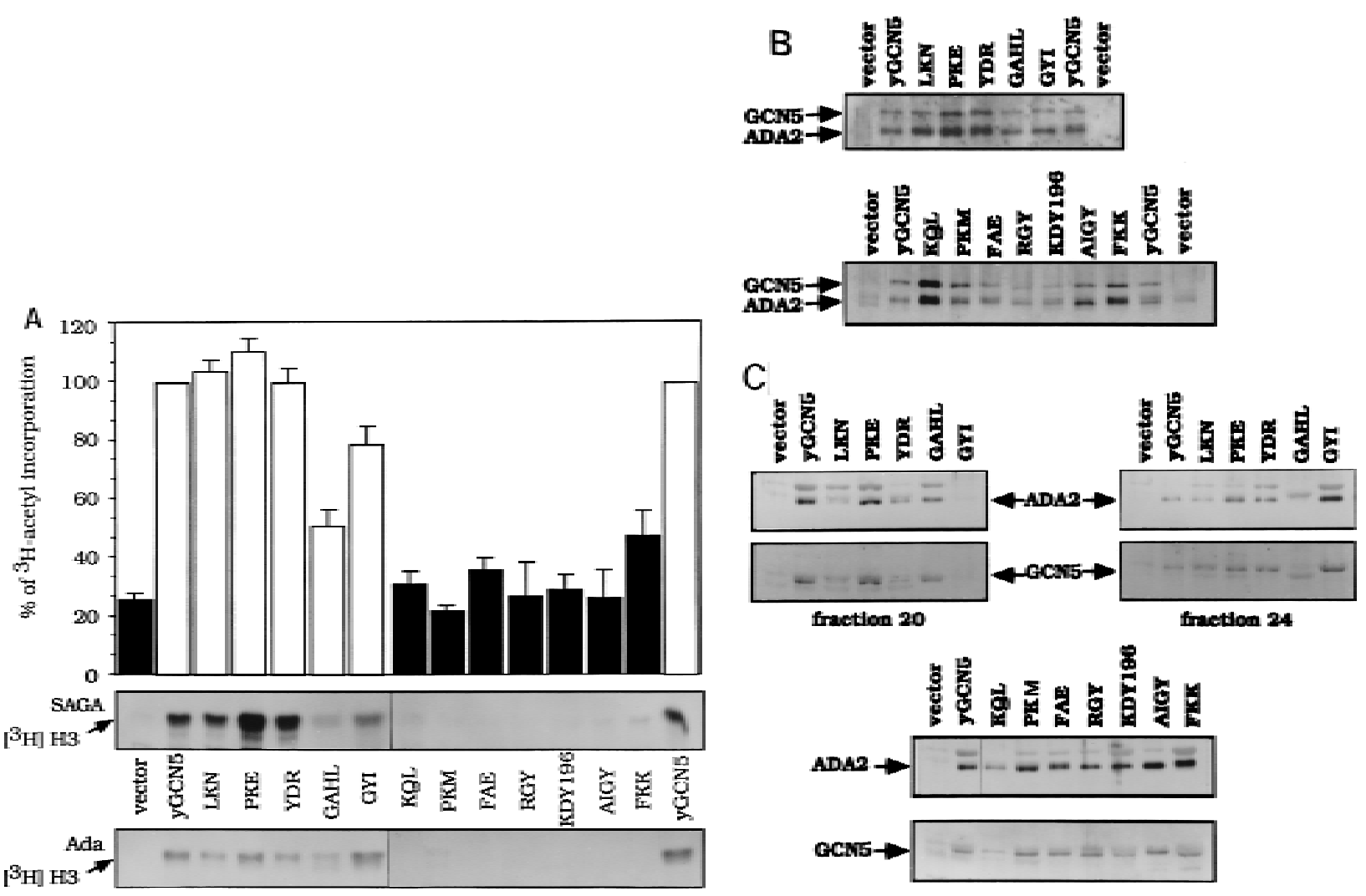

Figure 6. Free histone HAT activity of Gcn5 substitution mutants in SAGA and Ada complexes. (A) SAGA and Ada complexes prepared from Gcn5 mutants displaying wild-type activity in functional assays (light gray bars in Fig. 4) and Gcn5-defective mutants (solid bars in Fig. 4) were tested for HAT activity. SA GA and Ada complexes of each mutants containing peak Gcn5 and Ada2 proteins detected by Western blots $(B, C)$ were used. Complexes were incubated with free core histones and $\left[{ }^{3} \mathrm{H}\right]$ acetyl $\mathrm{CoA}$. Reaction mixtures were then subjected to liquid scintillation accounting (top) as well as SDS-PAGE and fluorography (middle and bottom). The activity from SAGA complexes (middle) and Ada complexes (bottom) are indicated by arrows. Quantitation of corresponding scintillation assays for SAGA is shown as a percentage of wild-type Gcn5. Error bars represent the standard error about the mean from three independent experiments. Western bl ots of Gcn5 and Ada2 proteins from SAGA (B) and Ada (C) complexes. Peak fractions from SAGA and $A$ da complexes were detected for the presence of $G \mathrm{cn} 5$ and $A d a 2$ using immunoblot analysis using Gcn5 and $A d a 2$ antisera.

somal histones. Because the level of nucleosomal acetylation compared with free histone acetylation is markedly inhibited in the SAGA complex (D. Sterner, P. Grant, J. Workman, and S.L. Berger, unpubl.), we tested the ability of the Gcn5 substitution mutants to acetylate nucleosomal histones in the context of the Ada complex. In addition, the Gcn5-dependent H3/H2B HAT activity in the Ada complex overlaps a Gcn5-independent $\mathrm{H} 4$ / H2A HAT activity (Grant et al. 1997; Fig. 7), which provides an internal control for variation in extract preparation.

Fractions between 18 and 30 were tested for HAT activity on nucleosomal substrates using a liquid HAT assay and incorporation of the $\left[{ }^{3} \mathrm{H}\right]$ acetyl group into histones was visualized by PAGE and fluorography. The peak of Gcn5-dependent H3/H2B activity in the wild-type extract eluted between fractions 2024 , whereas the $\mathrm{H} 4 / \mathrm{H} 2 \mathrm{~A}$ activity peaked at fraction 24-26 (Fig. 7, top left). As observed previously (Grant et al. 1997), in extracts prepared from the gcn5strain, the $\mathrm{H} 4 / \mathrm{H} 2 \mathrm{~A}$ HAT activity remained intact, whereas the H3/H2B activity disappeared (Fig. 7, left).

Strikingly, five mutants that failed to complement loss of Gcn5 in vivo (KQL, PKM, FAE, AIGY, and FKK) and were defective in acetylation of free histones in the SAGA and Ada complexes also were completely defective in acetylation of nucleosomal histones in the Ada complex (Fig 7, right). The remaining defective mutant (KDY 196) had severely reduced activity, which was barely above background levels present in the gcn5- extract (Fig. 7, right). In addition, one of the partially defective mutants in the in vivo assays (RGY) showed severely impaired activity in the nucleosome acetylation assay (Fig. 7, right), as also observed in the free histone assay (Fig. 6A). In contrast, the Gon5-independent $\mathrm{H} 4$ / H2A acetylation activity was not affected by these $\mathrm{Gcn} 5$ substitution mutations (Fig. 7, right). As described in the previous section, the presence of Gcn5 and Ada2 in the Ada complex was confirmed for all mutants by Western analysis (Fig 6C).

In contrast, mutants that displayed wild-type pheno- 
Figure 7. Nucleosomal HAT activities of Gcn5 substitution mutants in Ada complexes. The same wild-type mutants and defective mutants used in the free histone assays (Fig. 6) were subjected to nucleosomal HAT assays using Ada complexes. Even-numbered $\mathrm{M}$ ono $\mathrm{Q}$ column fractions from Ada complex of each mutant were incubated with oligonucleosome cores and $\left[{ }^{3} \mathrm{H}\right.$ ]acetyl CoA. Reactions were loaded onto SDS-PAGE and the gels were analyzed by fluorography. (Left) Wild-type Gcn5, vector al one, and wild-type Gcn5 substitution mutants; (right) defective Gcn5 mutants. Positions of core histones on the gel are indicated by arrows. Numbers at the top are the $\mathrm{M}$ ono $\mathrm{Q}$ column fractions. The $\mathrm{H} 3 / \mathrm{H} 2 \mathrm{~B}$ nucleosomal activity is Gcn5-dependent, and the $\mathrm{H} 4 / \mathrm{H} 2 \mathrm{~A}$ nucleosomal HAT activity is Gcn5-independent (Grant et al. 1997).

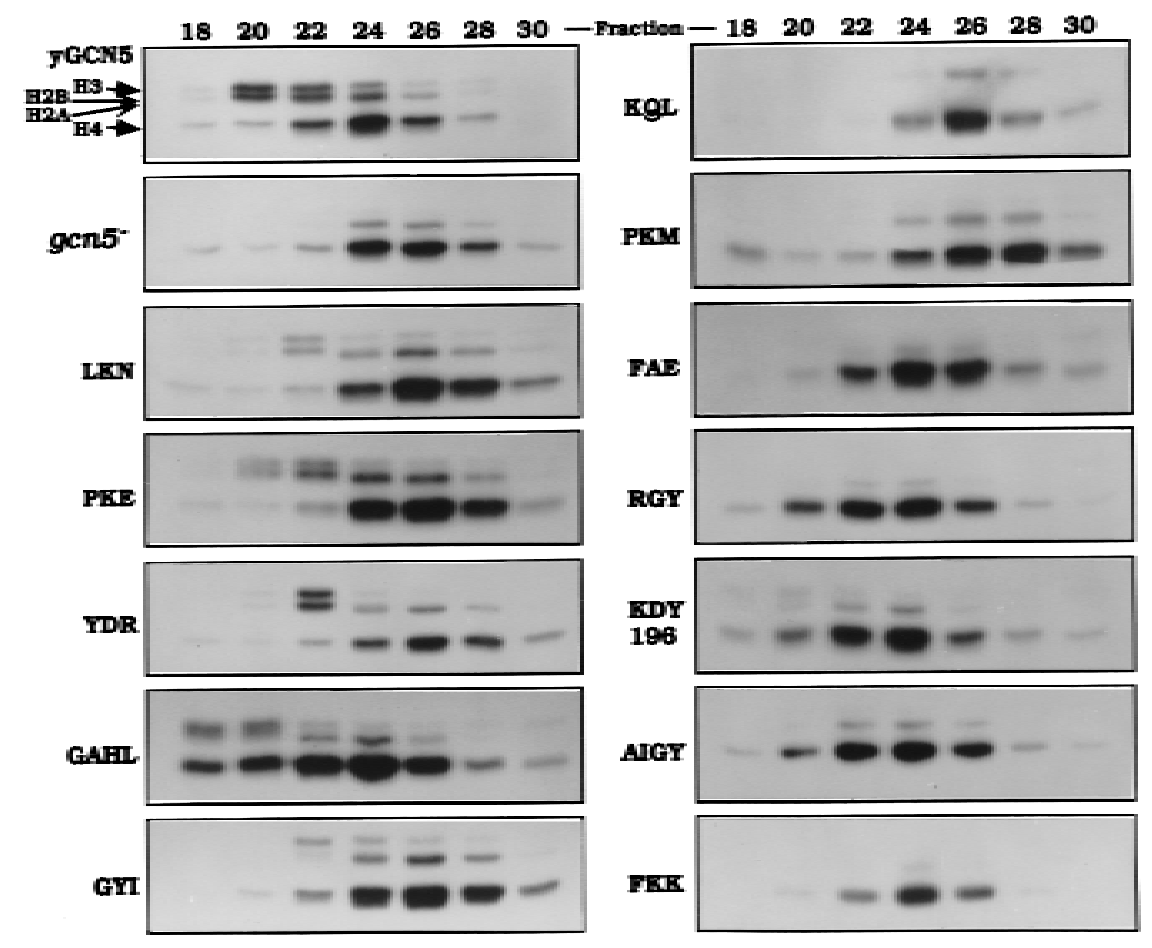

types in complementation of growth and transcription in the gen $5^{-}$strain (LKN , PKE, YDR, GAHL, and GYI), were competent for acetylation of nucleosomal $\mathrm{H} 3 / \mathrm{H} 2 \mathrm{~B}$ with varying efficiencies relative to wild-type Gcn5 (Fig. 7, left). Significantly, all wild-type Gcn5 mutants were relatively strong in nucleosomal $\mathrm{H} 3 / \mathrm{H} 2 \mathrm{~B}$ acetylation compared with all defective mutants. Therefore, the data demonstrate that mutations that are most deleterious for in vivo function of $\mathrm{Gcn} 5$ similarly completely impair its histone acetylation function as a component of native SAGA and Ada complexes.

\section{Discussion}

Several transcriptional cofactors have been revealed recently to possess histone acetyltransferase activity in vitro. These include members of the G cn5 family as well as the unrelated coactivators $\mathrm{p} 300 / \mathrm{CBP}$ and $\mathrm{TAF}_{11} 250$. The emergence of this new class of transcription coactivators functioning as HATs has raised important questions. First, do these proteins function as acetyltransferases in vivo, second, are nucleosomal histones their natural substrates, and, finally, is acetyltransferase activity required for transcription function? To address these issues, we systematically created substitution mutants in the putative HAT domain of yeast Gcn5, and compared their effects on growth and transcriptional activation in vivo with histone acetylation in vitro. If acetylation of histones by Gcn5 is critical, then there should be strong correlation between HAT activity and in vivo function.
Correlation between in vivo function of Gcn5 and in vitro histone acetylation

We identified six substitution mutations (KQL, PKM, FAE, KDY 196, AIGY, and FKK) in Gen5 that were highly deleterious to Gcn5 function in vivo. Strikingly, five were inert for acetylation of histones, either as free or nucleosomal substrates, and the sixth mutant (KDY 196) had very low activity. Importantly, other mutants that were intermediate in function in vivo (YIA, RGY, DNY, KDY 242, and TLM) were also impaired significantly in both free histone and nucleosomal histone acetylation (RGY acetylation is shown in Figs. 6 and 7; others not shown). Finally, substitution mutants that displayed wild-type function in vivo, displayed relatively high levels of acetylation in vitro (five examples are shown in Figs. 6 and 7; complexes from six others were prepared and were found to possess significant levels of HAT activity). Therefore, these data (summarized in Fig. 8) demonstrate a clear correlation between residues within Gcn5 that are critical for acetylation of histone substrates, and residues that are absolutely required for growth and transcriptional activity of Gcn5.

It is important to stress that we analyzed histone acetylation by $\mathrm{Gcn} 5$ within the context of native yeast SAGA and Ada complexes. Gcn5, as well as other HATs (Chen et al. 1997) and HDACs (Rundlett and Grunstein 1996), exists in protein complexes containing multiple components that, in the case of Gcn5, alter substrate specificity in two ways. Recombinant yeast Gcn5 acetylates only free histones and primarily histone $\mathrm{H} 3$ (histone $\mathrm{H} 4$ is acetylated to a far lesser degree) (Brownell et 


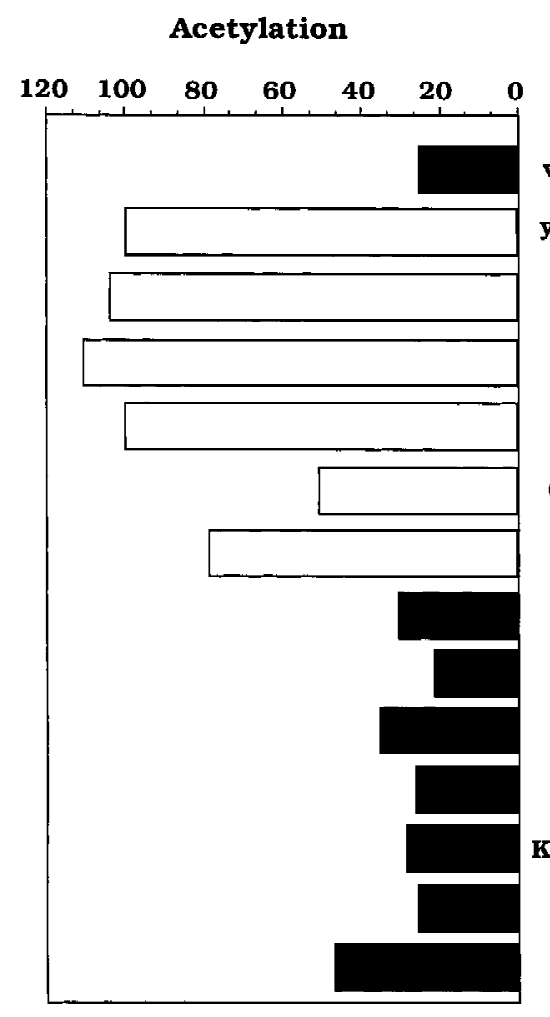

\section{Transcription}

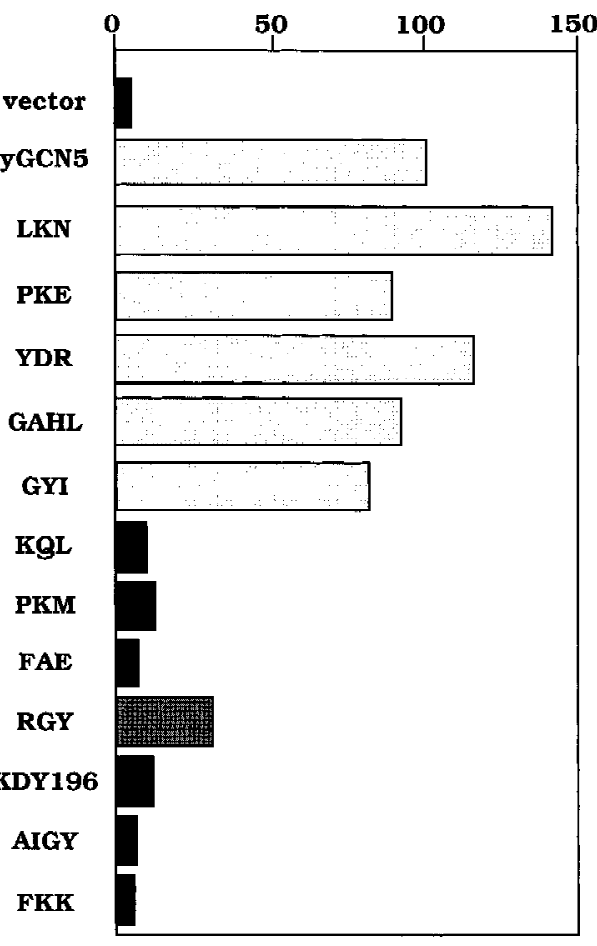

Figure 8. Comparison of mutant Gcn5 phenotypes in acetylation vs. transcription. Quantitative data are taken from Fig. $6 \mathrm{~A}$ (top) and Fig. 4 (the Gal4-VP16 and LexA-Gcn4 data were averaged). Acetylation of free histones by the SAGA complex is shown at left and transcriptional activation by the chimeric activators is shown at right. al. 1996). Gcn5 in native yeast SAGA and A da complexes acetylates both free and nucleosomal histones and targets both $\mathrm{H} 3$ and H2B (Grant et al. 1997). The altered specificity of Gcn5 in complexes, as opposed to recombinant form, suggests that physiologically relevant acetylation activity is best evaluated in the context of these native HAT complexes, as we have done in the current study.

Mutations that impair transcription similarly impair growth

Gcn5 mutants were tested for their effects on transcription using two heterologous model activators, Gal4VP16 and LexA-Gcn4. We showed previously that Ada2, which is present in the Ada and SA GA complexes and is required for $\mathrm{Gcn} 5$ function in vivo, binds to both of these activation domains (Barlev et al. 1995). The Gcn5 substitution mutants showed very similar phenotypes in the transcription assays, such that the wild-type, intermediate, and del eterious mutants displ ayed paral lel effects for function by each activator. In addition, the natural HIS3 and $\mathrm{PHO} 5$ promoters also display dependency on the defective mutants, and have normal function in the presence of wild-type mutants (R. Bol otserkovskaya, N . Barlev, and S.L. Berger, unpubl.). Therefore, the effects of the substitution mutations that reduce histone acetylation have similar effects on both synthetic model promoters and natural yeast promoters, arguing that the effects are generally consistent for activators that require Gcn5 for function.

M oreover, we found a striking correlation between the effect of the substitution mutations on Gcn5 function in growth and in transcriptional activation assays, such that colony size and doubling time of the wild-type, intermediate, and deleterious mutants mirrored transcription function. The consistency between growth and transcription suggests that results obtained using the model activators Gal 4-VP16 and LexA-Gcn4 reflect natural dependency of yeast genes transcription on Gcn5.

Taken together with previous data regarding function and biochemical interactions of the Ada, Gcn5, and Spt proteins (Grant et al. 1997; Roberts and Winston 1997), the data support the model that Gcn5 functions within Ada and/or SAGA complexes to acetylate nucleosomal histones in promoter regions of dependent genes. Ada and SAGA may be recruited to promoters via interactions of Ada2 or Gcn5 with activation domains (Silverman et al. 1994; Barlev et al. 1995; Chiang et al. 1996), which may position the HAT domain to acetylate nucleosomes proximal to the TATA box. Spt proteins may have distinct roles in the SA GA complex, either for complex integrity (Spt7 and Spt20/Ada5; Grant et al. 1997) or recruitment of TBP and associated proteins (Spt7 and Spt8; Eisenmann et al. 1992, 1994). Based on the strong correlation we observe between residues within Gcn5 required for HAT activity and function, we conclude that acetylation by Ada and/or SAGA appears to be a critical in vivo function.

Residues important for function within the Gen5 HAT domain

We identified critical residues for function in all four 
conserved subdomains of Gcn5. Mutations in regions I (KQL and PKM), II (FAE and RGY), and III (AIGY and FKK) were most debilitating for histone acetylation within the Ada and SAGA complexes. Previous evolutionary comparisons have identified conserved sequences in the putative HAT domain between the Gcn5 subfamily of acetyltransferases and many additional proteins, leading to speculation that these proteins form an extensive superfamily (Reifsnyder et al. 1996; N euwald and Lansman 1997). In particular, there is very strong conservation of conserved Gcn5 regions II and III and members of the superfamily (N euwald and Lansman 1997). Because the apparent commonal ity within the superfamily is acetyl-CoA binding, this region may bind acetyl-CoA. In particular, RGY, AIGY, and FKK show very strong conservation across the superfamily. Therefore, our results that these mutations are extremely deleterious to histone acetylation are consistent with a role in acetyl-CoA binding. Whereas AIGY and FKK are completely debilitating to function of Gcn5 in vivo, it was surprising that RGY mutant was not affected as dramatically. Because the two glycines in RGYG show the most strong conservation in this short sequence ( $N$ euwal $d$ and Lansman 1997), it may be that the al anine substitution preserves some function. The dominant negativity of all three of these mutants RGY, AIGY, and FKK supports the interpretation of a defect in acetyl-CoA binding, but retention of other critical protein interactions.

Interestingly, the FAE mutant, which occurs at the amino-terminal border of region $\mathrm{II}$, is one of the most dysfunctional mutants, but is not strongly conserved in the superfamily. The biochemical function of the FAE residues will be important to determine. In addition, another region of conservation in the superfamily, located between regions I and II, contains the conserved GGI (N euwald and Lansman 1997). In our study, the GGI mutant was not affected in vivo, but, as is true for the RGYG mutant, the alanine substitutions may not be profound sequence changes for these particular resi dues. Overall, our data support previous speculation that regions II and III are involved in acetyl-CoA binding.

Regions I and IV of the Gcn5 subfamily display no significant homology to the other members of the superfamily (N euwal d and Lansman 1997). TheKQL and PKM mutations in region I, however, result in similar phenotypes as the mutations in the putative acetyl-CoA-binding site in regions II and III. These phenotypes include loss of histone acetyltransferase activity, and loss of growth and transcription function in vivo, as well as strong dominant negativity. One possibility is that region I is involved in binding of the particular substrates acetylated by Gcn5. If this is true, then the lack of homology in region I within the superfamily would imply that the other family members acetylate substrates other than nucleosomal histones $\mathrm{H} 3$ and $\mathrm{H} 2 \mathrm{~B}$. These alternate substrates could include free histones, histones $\mathrm{H} 4$ or $\mathrm{H} 2 \mathrm{~A}$, or completely dissimilar acetylation targets.

The analysis of mutants KDY 196 and KDY 242 suggests an important role for these residues in processes other than direct nucleosome acetylation. Both mutants had extremely debilitated function in vivo, but retained Iow acetylation function (see Fig. 7 for KDY 196; data not shown for KDY242). These phenotypes suggest a possible regulatory function for these resi dues or interaction with other proteins that are not involved in acetylation. In particular, because region IV is not well conserved with other acetyltransferases (N euwald and Lansman 1997), and KDY 242 was the only profoundly defective mutant in this region, this is consistent with a function of region IV specific to regulation of Gcn5.

Overall, the data demonstrate that all four highly conserved subdomains contain important el ements for function of Gcn5. Regions I, II, and III are likely to be involved in enzymatic activity and substrate specificity, whereas region IV may have a regulatory role.

Are histones bona fide physiological substrates of Gcn5?

As noted above, the HAT domains of Gcn5 subfamily members, although remarkably conserved with respect to each other, display no obvious sequence conservation with the putative HAT domains of either CBP/p300 or $\operatorname{TAF}_{11} 250$ (nor are the latter two HAT domains mutually conserved). These observations raise the possibility that the known HATs share yet unidentified structural motifs. A second, and not exclusive explanation is that significant differences exist in the substrates of acetylation by the Gcn5 subfamily compared with the other putative acetyltransferases. Notably, p300 has been shown recently to acetylate certain lysines in the carboxyl terminus of the tumor suppressor and activator p53 (Gu and Roeder 1997).

Our data are consistent with the hypothesis that nucleosomal histones are critical physiological substrates of the Gcn5 family. We scanned the conserved residues of the entire HAT domain, and observed a strong consistency between the effect of the most deleterious $\mathrm{G}$ cn5 substitution mutants on function in vivo and their effect on histone acetylation in vitro. Moreover, recent data indicate that normal remodeling of nucleosomes at the PHO5 promoter during transcriptional activation requires $\mathrm{Gcn} 5$, and in particular, the remodeling mirrors the pattern of dependency on the HAT domain mutations depicted here ( $P$. Gregory, A. Schmid, M. Zavari, L. Liu, S.L. Berger, and W. Hörz, unpubl.). Taken together the data strongl y suggest that histones are among the key substrates of Gcn5 in vivo.

\section{Materials and methods}

Yeast strains

The yeast strain PSY 316 (MAT $\alpha$ ade-101 $\Delta$ his3-200 leu2-3,112 lys2 ura3-52) and its derivatives PSY $316 \Delta$ gen5 (Candau and Berger 1996) were used for transformation of HAT domain substitution mutations in conserved regions II, III, and IV. The TRP1 disruption of IPY $3 \Delta$ gcn 5 (Roberts and Winston 1997) was constructed using the hisgU RA 3 cassette as described (Alani et al. 1987), and was used for integration of the HAT domain substitution mutations in conserved region I. 


\section{Plasmids}

pRS414-GCN5 was made by amplifying GCN5 including its natural promoter and terminator from genomic DNA using PCR with primers containing $5^{\prime}$-Xhol $\rightarrow$ 3'-Bglll sites and then cloning GCN5 into the low-copy yeast expression vector pRS414 at Xhol and BamHI sites. GCN5 with Bglll ends was ligated into high-copy yeast gal actose-inducible expression vector pRS424 $\left(\mathrm{P}_{\mathrm{Gal}}\right)$ to produce pRS424( $\left.\mathrm{P}_{\mathrm{Gal}}\right)-\mathrm{GCN} 5$. GCN5 was mutagenized in pRSET, pRS414 or pRS424( $\left.\mathrm{P}_{\mathrm{Gal}}\right)$ backbone and then subcloned into other vectors by exchanging BamHI-EcoRI (for subregion I) or N col (subregi on II-IV) fragments. GCN 5 subregion I mutants were subcloned into pRS306 for integration. The yeast-inducible expression vector containing GST was made by inserting GST fragment (amplified by PCR from pGEX plasmid with BamHI and $\mathrm{Kpnl}$ sites) into the yeast galactose inducible expression vector pYES. GST-ADA2 was made by inserting ADA2 at BgllI in frame with GST.

\section{Site-directed mutagenesis}

The Chameleon double-stranded site-directed mutagenesis kit (Stratagene) was used to construct the al anine-scan mutants of Gcn5 using mutagenic oligonucleotides shown in Table 1. Briefly, the GCN5 plasmid was heat-denatured and hybridized with two oligonucleotide primers. The selection primer changed one nonessential unique restriction site to a new restriction site on the plasmid backbone. The second primer encoded the specific mutations, in which three or four amino acids in Gcn5 were changed into three or four al anine residues, and a new Pstl restriction site was created simultaneously. Unmutagenized parental plasmids were selected against by digestion using the mutagenized restriction site in the vector backbone, and mutagenized progeny were identified using the introduced Pstl site and were confirmed by sequencing.

\section{Growth and dominant-negative assays}

Growth phenotypes of Gcn5 substitution mutations were analyzed after transformation of IPY $37 \Delta$ gen $5 \Delta \operatorname{trpl}$ (conserved region I mutations) PSY $316 \Delta$ gen5 $5 \Delta$ trpl (conserved regions II-IV mutations). Single colonies were streaked on minimal synthetic medium, and were incubated at $30^{\circ} \mathrm{C}$ for 2 days. For the doubling time assay, single colonies were inoculated into liquid fully supplemented synthetic media and rotated overnight at $30^{\circ} \mathrm{C}$. The cultures were then diluted to an $A_{600}$ of 0.02 , and the OD was checked every $2 \mathrm{hr}$ up to a total of $28 \mathrm{hr}$.

Dominant-negative assays were carried out by transforming high-copy expression plasmids pRS425( $\left.\mathrm{P}_{\mathrm{Gal}}\right)$ or pRS424( $\left.\mathrm{P}_{\mathrm{Gal}}\right)$ containing GCN 5 mutants into IPY 36 or PSY 316 strains bearing wild-type GCN5. Transformants were plated on SD (2\% glucose) plates, incubated at $30^{\circ} \mathrm{C}$ for 3 days. Single colonies were restreaked on SD ( $2 \%$ galactose) medium, and incubated at room temperature for 3 days.

\section{Transcription assays}

Transcription assays were performed in IPY $37 \Delta$ gen $5 \Delta$ trpl (conserved region I mutations) and PSY $316 \Delta$ gen5 4 trpl (conserved regions II-IV mutations). Plasmids expressing Gal4-VP16 ${ }_{\mathrm{FA}}$ (Berger et al. 1990) and LexA-Gcn4 (M arcus et al. 1994) and the corresponding reporters were cotransformed. $\beta-G$ al activity was determined as units per milligram of protein. The LacZ reporters used were pLGSD5 (Guarente et al. 1982) for Gal4-VP16 activation, and LexA-8x (eight LexA-binding sites) (Candau et al. 1996) for LexA-Gcn4 activation.
Yeast protein overexpression and in vivo GST-binding assay

Yeast PSY 316 $\Delta$ gen5 strain transformed with GST-ADA2 and pRS424( $\left.P_{G \text { al }}\right)-G C N 5$ or GCN5 substitution mutants were inoculated into $5 \mathrm{ml}$ of synthetic glucose media and grown overnight. The cultures were diluted in $100 \mathrm{ml}$ of synthetic media containing 2\% lactate, and grown for 5-6 hr. Galactose was added to $2 \%$ final concentration to induce protein expression. Cells were harvested when cultures reached $A_{600}=1$. Cell pellets were washed and resuspended in $400 \mu \mathrm{l}$ of storage buffer (20 mM HEPES at pH 8.0, $5 \mathrm{~nm}$ EDTA, 20\% glycerol, $1 \mathrm{~mm} \beta$-mercaptoethanol, 1 M PMSF). Cells were disrupted by vortexing with glass beads and supernatants were collected. This extract (200 $\mu \mathrm{l}$ ) was incubated with $100 \mu \mathrm{l}$ of GST bead slurry (50\%) for $1 \mathrm{hr}$ in PBS buffer-0.1\% Triton-100, and beads were washed extensively with PBS buffer. The proteins bound to GST beads were analyzed by SDS-PAGE and immunoblotted using Gcn5 antiserum (Candau and Berger 1996).

\section{Yeast protein complex preparation}

Strains transformed with GCN5 substitution mutants were grown selectively in $200 \mathrm{ml}$ of synthetic media, and then transferred to 4 liters of YPD media and grown to $A_{600}=2$. Cells were harvested and resuspended in $30 \mathrm{ml}$ of extraction buffer $(40 \mathrm{~mm}$ HEPES at pH 7.3, $350 \mathrm{~mm} \mathrm{NaCl}, 0.1 \%$ Tween, 10\% glycerol, protease inhibitors). Cells were lysed by glass beads using a bead beater, and extracts were clarified by centrifugation for $1 \mathrm{hr}$ at $40,000 \mathrm{~g}$. N ext, extracts were incubated with $10 \mathrm{ml} \mathrm{Ni-NTA-}$ agarose slurry $(50 \%)$ at $4^{\circ} \mathrm{C}$ for $2.5 \mathrm{hr}$. Resins were poured into a column and washed subsequently with $10 \mathrm{ml}$ of extraction buffer, $10 \mathrm{ml}$ of buffer A (20 mM Imidazole at pH 7.0, $100 \mathrm{~mm}$ $\mathrm{NaCl}, 0.1 \%$ Tween $20,10 \%$ glycerol, protease inhibitors). Bound proteins were eluted with $10 \mathrm{ml}$ of buffer A with $300 \mathrm{~mm}$ imidazole. The $300 \mathrm{~mm}$ imidazole eluate was directly loaded onto a M ono Q HR5/5 column equilibrated in buffer B (50 mM Tris at $\mathrm{pH} 8.0,100 \mathrm{~mm} \mathrm{NaCl}, 0.1 \%$ Tween 20, 10\% glycerol). After a 10-ml wash with buffer $\mathrm{B}$, bound proteins were eluted with a $25-\mathrm{ml}$ linear gradient of $100 \mathrm{~mm}$ to $500 \mathrm{~mm} \mathrm{NaCl}$ in buffer B. Fractions were collected and subjected to HAT assay.

\section{Western blot analysis}

The substitution mutations in the HAT domain of Gen5 unpredictably altered the elution position of the SAGA complex from the M ono Q column, and therefore the peak fraction of SAGA elution for each mutant was determined by Western blot analysis using both Ada2 and Gcn5 antisera (data not shown). Then final Western blot analyses were performed with this peak fraction from the MonoQ column (Fig. 6B). For the Ada complex, peak fractions for protein analysis were fractions 20 or 24; fraction 22 could not be used because of a strong nonspecific crossreacting protein (data not shown). Western blot analyses were performed with Gcn5 and Ada2 antisera (Barlev et al. 1995; Candau and Berger 1996).

\section{HAT assays for free and nucleosomal histones}

Different amount of fractions ( 2 and $6 \mu$ ) were used for the nucleosomal HAT assay and similar histone acetylation patterns $(\mathrm{H} 3 / \mathrm{H} 2 \mathrm{~B}$ relative to $\mathrm{H} 4 / \mathrm{H} 2 \mathrm{~A}$ ) were observed (data not shown), indicating that the amount of extracts used in the HAT assay was in the linear range of HAT enzymatic activity. For nucleosomal HAT assays, M onoQ fractions from Ada complex of each Gcn 5 mutant were used. Three microliters of each even numbered fraction (from 18 to 30 ) were incubated with $1 \mu \mathrm{g}$ of 
oligonucleosome cores and $\left[{ }^{3} \mathrm{H}\right]$ acetyl $\mathrm{COA}$ in HAT buffer (Grant et al. 1997) at $30^{\circ} \mathrm{C}$ for $30 \mathrm{~min}$. The reactions were subjected to SDS-PAGE, and gels were then treated with Intensify Buffer (DuPont NEN) and fluorographed. For free histone HAT assays, the same fractions of SAGA and Ada complexes used in Western analysis were incubated with $1 \mu \mathrm{g}$ core histones and $\left[{ }^{3} \mathrm{H}\right.$ ]acetyl COA in HAT buffer. Reactions and fluorograph were carried out as indicated above.

\section{Acknowledgments}

We are grateful to R. M armorstein for preparation of Figure $1 \mathrm{~A}$. We thank C.D. Allis and M.H. Kuo for communication of unpublished results. We thank J. Workman for oligonucleosomes and D. Sterner and F. Winston for yeast strains. We thank P. Lieberman, G. M oore, F. Rauscher, G. Rovera, and J. Workmen for support and valuable discussions; N . Barlev and G. M oore for critical reading of the manuscript; and A. Kulak for expert editorial help. The work was supported by grants from the $\mathrm{Na}$ tional Science Foundation and The Council for Tobacco Research to S.L.B; S.L.B is the recipient of an American Cancer Society Junior Faculty Research Award.

The publication costs of this article were defrayed in part by payment of page charges. This article must therefore be hereby marked "advertisement" in accordance with 18 USC section 1734 solely to indicate this fact.

\section{References}

Alani, E., L. Cao, and N. Kleckner. 1987. A method for gene disruption that allows repeated use of URA 3 sel ection in the construction of multiply disrupted yeast strains. Genetics 116: 541-545.

Alland, L., R. Muhle, H. Hou Jr., J. Potes, L. Chin, N. SchreiberAgus, and R.A. DePinho. 1997. Role for N-CoR and histone deacetylase in Sin3-mediated transcriptional repression. $\mathrm{Na}$ ture 387: 49-55.

Bannister, A. and T. Kouzarides. 1996. The CBP co-activator is a histone acetyltransferase. Nature 384: 641-643.

Barlev, N., R. Candau, L. Wang, P. Darpino, N. Silverman, and S. Berger. 1995. Characterization of physical interactions of the putative transcriptional adaptor, ADA2, with acidic activation domains and TATA-binding protein. J. Biol. Chem. 270: 19337-19344.

Berger, S.L., W.D. Cress, A. Cress, S.J. Triezenberg, and L. Guarente. 1990. Selective inhibition of activated but not basal transcription by the acidic activation domain of VP16: Evidence for transcriptional adaptors. Cell 61: 1199-1208.

Berger, S.L., B. Pina, N. Silverman, G.A. M arcus, J. A gapite, J.L. Regier, S.J. Triezenberg, and L. Guarente. 1992. Genetic isolation of ADA2: A potential transcriptional adaptor required for function of certain acidic activation domains. Cell 70: 251-265.

Bradbury, E.M. 1992. Reversible histone modifications and the chromosome cell cycle. BioEssays 14: 9-16.

Brownell, J., J. Zhou, T. Ranalli, R. Kobayashi, D. Edmondson, S. Roth, and C. D. Allis. 1996. Tetrahymena histone acetyltransferase A: A transcriptional co-activator linking gene expression to histone acetylation. Cell 84: 843-851.

Candau, R. and S.L. Berger. 1996. Structural and functional analysis of yeast putative adaptors: Evidence for an adaptor complex in vivo. J. Biol. Chem. 271: 5237-5345.

Candau, R., P. Moore, L. Wang, N. Barlev, C. Ying, C. Rosen, and S. Berger. 1996. Identification of functionally conserved human homologues of the yeast adaptors ADA 2 and GCN 5 . Mol. Cell. Biol. 16: 593-602.
Candau, R., J. Zhou, C.D. Allis, and S.L. Berger. 1997. Histone acetyltransferase activity and interaction with ADA2 are critical for GCN 5 function in vivo. EMBO J. 16: 555-565.

Chen, H., R. Lin, R. Schiltz, D. Chakravarti, A. N ash, L. N agy, M. Privalsky, Y. Nakatani, and R. Evans. 1997. Nuclear receptor coactivator ACTR is a novel histone acetyltransferase and forms a multimeric activation complex with PCAF and CBP/p300. Cell 90: 569-580.

Chiang, Y., P. Komarnitsky, D. Chase, and C. Denis. 1996. ADR 1 activation domains contact the histone acetyltransferase GCN 5 and the core transcriptional factor TFIIB. J. Biol. Chem. 271: 32359-32365.

Cote, J., J. Quinn, J.L. Workman, and C.L. Peterson. 1994 Stimulation of GAL4 derivative binding to nucleosomal DNA by the yeast SWI/SN F complex. Science 265: 53-60.

Eisenmann, D., K. Arndt, S. Ricupero, J. Rooney, and F. Winston. 1992. SPT 3 interacts with TFIID to allow normal transcription in Saccharomyces cerevisiae. Genes \& Dev. 6: 1319-1331.

Eisenmann, D., C. Chapon, S. Roberts, C. Dollard, and F. Winston. 1994. The Saccharomyces cerevisiae SPT 8 gene encodes a very acidic protein that is functionally related to SPT 3 and TATA-binding protein. Genetics 137: 647-657.

Fascher, K., J. Schmitz, and W. Horz. 1993. Structural and functional requirements for the chromatin transition at the $\mathrm{PHO} 5$ promoter in Saccharomyces cerevisiae upon $\mathrm{PHO} 5$ activation. J. Mol. Biol. 231: 658-667.

Gansheroff, L., C. Dollard, P. Tan, and F. Winston. 1995. The S. cerevisiase SPT7 gene encodes a very acidic protein important for transcription in vivo. Genetics 139: 523-536.

Georgakopoulos, T. and G. Thireos. 1992. Two distinct yeast transcriptional activators require the function of the GCN 5 protein to promote normal levels of transcription. EMBO J. 11: $4145-4152$.

Gill, G. and M. Ptashne. 1988. N egative effect of the transcriptional activator GAL4. Nature 334: 4721-4724.

Grant, P.A., L. Duggan, J. Cote, S.M. Roberts, J. Brownell, R. Candau, R. Ohba, T. Owen-Hughes, C.D. Allis, F. Winston, S.L. Berger, and J.L. Workman. 1997. yGCN 5 function within multisubunit ADA and SPT/ADA adaptor complexes to acetylate nucleosomal histones. Genes \& Dev. 11: $1640-1650$.

Grunstein, M. 1990. Nucleosomes: regulators of transcription. Trends Genet. 6: 395-400.

Gu, W. and R. Roeder. 1997. Activation of p53 sequence-specific DN A binding by acetylation of the p53 C-terminal domain. Cell 90: 595-606.

Guarante, L. 1995. Transcriptional coactivators in yeast and beyond. Trends Biol. Sci. 20: 517-521.

Guarente, L., R.R. Yocum, and P. Gifford. 1982. A GAL10CYC1 hybrid yeast promoter identifies the GAL4 regulatory region as an upstream site. Proc. Natl. Acad. Sci. 79: 74107414.

Hassig, C.A., T.C. Fleischer, A.N . Billin, S.L. Schreiber, and D.E. Ayer. 1997. Histone deacetylase activity is required for full transcriptional repression by $\mathrm{mSin3A}$. Cell 89: 341-347.

Heinzel, T., R.M . Lavinsky, T.M. Mullen, M. Soderstrom, C.D. Laherty, J. Torchia, W.M. Yang, G. Brard, S.D. N go, J.R. Davie, E. Seto, R.N. Eisenman, D.W. Rose, C.K. Glass, and M.G. Rosenfeld. 1997. A complex containing N-CoR, mSin3 and histone deacetylase mediates transcriptional repression [see comments]. Nature 387: 43-48.

Kadosh, D. and K. Struhl. 1997. Repression by Ume6 involves recruitment of a complex containing Sin3 corepressor and Rpd3 histone deacetylase to target promoters. Cell 89: 365371. 
Kingston, R.E., C.A. Bunker, and A.N . Imbalzano. 1996. Repression and activation by multiprotein complexes that alter chromatin structure. Genes \& Dev. 10: 905-920.

Laherty, C.D., W.M. Yang, J.M. Sun, J.R. Davie, E. Seto, and R.N. Eisenman. 1997. Histone deacetylases associated with the mSin3 corepressor mediate mad transcriptional repression. Cell 89: 349-356.

Lee, D., J. Hayes, D. Pruss, and A. Wolffe. 1993. A positive role for histone acetylation in transcription factor access to nucleosomal DNA. Cell 72: 73-84.

Loidl, P. 1994. Histone acetylation: Facts and questions. Chromosoma 103: 441-449.

Marcus, G., N. Silverman, S. Berger, J. Horiuchi, and L. Guarente. 1994. Functional similarity and physical association between GCN 5 and ADA2 - putative transcriptional adaptors. EMBO J. 13: 4807-4815.

Marcus, G., J. Horiuchi, N. Silverman, and L. Guarente. 1996. ADA5/SPT 20 links the ADA and SPT genes, which are involved in yeast transcription. Mol. Cell. Biol. 16: 3197-3205.

Mizzen, C., X.-J. Yang, T. Kokubo, J. Brownell, A. Bannister, T. Owen-Hughes, J. Workman, L. Wang, S.L. Berger, T. Kouzarides, Y. N akatani, and C.D. Allis. 1996. The TAF250 Subunit of TFIID Has Histone Acetyltransferase Activity. Cell 87: 1261-1270.

Neuwald, A.F. and D. Lansman. 1997. GCN 5-related histone $\mathrm{N}$-acetyltransferases bel ong to a diverse superfamily that includes the yeast SPT10 protein. Trends Biochem. Sci. 22: 154-155.

Ogryzko, V., R. Schlitz, V. Russanova, B. Howard, and Y. N akatani. 1996. The Transcriptional Coactivators p300 and CBP are Histone Acetyltransferases. Cell 87: 953-959.

Owen-Hughes, T. and J.L. Workman. 1994. Experimental analysis of chromatin function in transcription control. Crit. Rev. Eukary. Gene Exp. 4: 403-441.

Paranjape, S.M., R.T. Kamakaka, and J.T. Kadonaga. 1994. Role of chromatin structure in the regulation of transcription by RN A polymerase II. Annu. Rev. Biochem. 63: 265-297.

Pazin, M. and J. Kadonaga. 1997. What's up and down with histone deacetylation and transcription? Cell 89: 325-328.

Peterson, C.L., A. Dingwall, and M.P. Scott. 1994. Five SWI/ SN F gene products are components of a large multisubunit complex required for transcriptional enhancement [see comments]. Proc. Natl. Acad. Sci. 91: 2905-2908.

Piña, B., S. Berger, G.A. M arcus, N. Silverman, J. A gapite, and L. Guarente. 1993. ADA3: A gene, identified by resistance to GAL4-VP16, with properties similar to and different from those of ADA2. Mol. Cell. Biol. 13: 5981-5989.

Reifsnyder, C., J. Lowell, A. Clarke, and L. Pillus. 1996. Yeast SAS silencing genes and human genes associated with $A M L$ and HIV-1 Tat interactions are homologous with acetyltransferases. Nature Genet. 14: 44-49.

Roberts, S. and F. Winston. 1996. SPT 20/ADA 5 encodes a novel protein functionally related to the TATA-binding protein and important for transcription in Saccharomyces cerevisiae. Mol. Cell. Biol. 16: 3206-3213.

_-_. 1997. Essential functional interactions of SAGA, a Saccaromyces cerevisiae complex of Spt, Ada and GCN 5 proteins, with the Snf/Swi and Srt/mediator complexes. Genetics 147: 451-465.

Rundlett, S.E. and M. Grunstein. 1996. HDA1 and RPD3 are members of distinct yeast histone deacetylase complexes that regulate silencing and transcription. Proc. Natl. Acad. Sci. 93: 14503-14508.

Sal eh, A., V. Lang, R. Cook, and C. Brand. 1997. Identification of native complexes containing the yeast coactivator-repressor proteins NGG1-ADA3 and ADA2. J. Biol. Chem. 272:
5571-5578.

Silverman, N., J. Agapite, and L. Guarente. 1994. Yeast ADA2 protein binds to the VP16 protein activation domain and activates transcription. Proc. Natl. Acad. Sci. 91: 11665-11668.

Struhl, K. 1995. Yeast transcriptional regulatory mechanisms. Annu. Rev. Genet. 29: 651-674.

Svaren, J., J. Schmitz, and W. Horz. 1994. The transactivation domain of $\mathrm{Pho} 4$ is required for nucleosome disruption at the PHO5 promoter. EMBO J. 13: 4856-4862.

Taunton, J., C.A. Hassig, and S.L. Schreiber. 1996. A mammalian histone deacetylase related to the yeast transcription regulator Rpd3p. Science 272: 408-411.

Triezenberg, S.J. 1995. Structure and function of transcriptional activation domains. Curr. O pin. Genet. Dev. 5: 190-196.

Turner, B.M. and L.P. O'N eill. 1995. Histone acetylation in chromatin and chromosomes. Sem. Cell Biol. 6: 229-236.

Vettese-Dadey, M., P.A. Grant, T.R. Hebbes, C. Crane-Robinson, C.D. Allis, and J.L. Workman. 1996. Acetylation of histone $\mathrm{H} 4$ plays a primary role in enhancing transcription factor binding to nucleosomal DN A in vitro. EMBO J. 15: 25082518.

Vidal, M. and R. Gaber. 1991. RPD3 encodes a second factor required to achieve maximum positive and negative transcriptional states in S. cerevisiae. Mol. Cell. Biol. 11: 63176327.

Wade, P. and A. Wolffe. 1997. Histone acetyltransferases in control. Curr. Biol. 7: 82-84.

Wang, L., C. Mizzen, C. Ying, R. Candau, N. Barlev, J. Brownell, C.D. Allis, and S. Berger. 1997. Histone Acetyltransferase activity is conserved between yeast and human GCN 5 and required for complementation of growth and transcriptional activation. Mol. Cell. Biol. 17: 519-527.

Wolffe, A. 1992. Chromatin: Structure and function. Academic Press, London, UK.

Yang, X., V. Ogryzko, J. Nishikawa, B. Howard, and Y. N akatani. 1996. A p300/CBP-associated factor that competes with the adenoviral E1A oncoprotein. Nature 382: 319-324.

Zawel, L. and D. Reinberg. 1995. Common themes in assembly and function of eukaryotic transcription complexes. Annu. Rev. Biochem. 64: 533-561.

Zhang, Y., R. Iratni, H. Erdjument-Bromage, P. Tempst, and D. Reinberg. 1997. Histone deacetylases and SAP18, a novel polypeptide, are components of a human Sin3 complex. Cell 89: 357-364. 


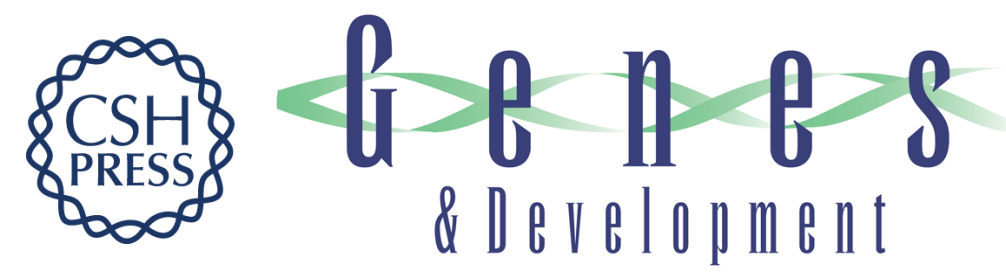

\section{Critical residues for histone acetylation by Gcn5, functioning in Ada and SAGA complexes, are also required for transcriptional function in vivo}

Lian Wang, Lin Liu and Shelley L. Berger

Genes Dev. 1998, 12:

References This article cites 57 articles, 24 of which can be accessed free at: http://genesdev.cshlp.org/content/12/5/640.full.html\#ref-list-1

License

Email Alerting Receive free email alerts when new articles cite this article - sign up in the box at the top Service right corner of the article or click here.

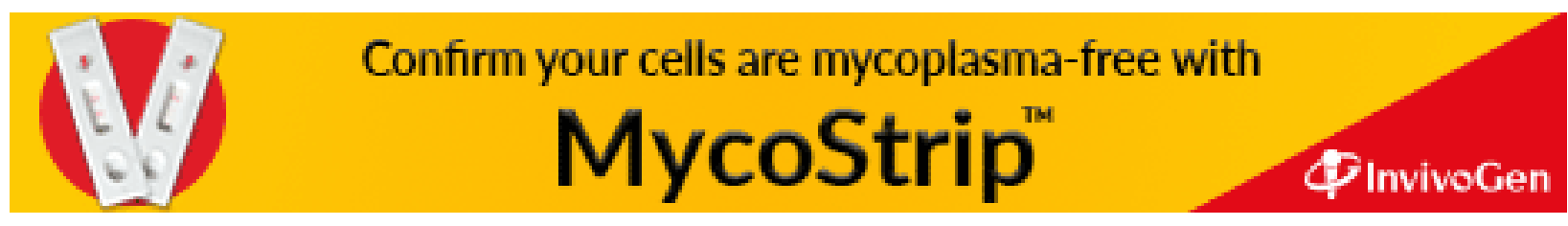

